\title{
Sex Differences in the Representation of Visuospatial Functions in the Human Brain
}

\author{
David W. Desmond \\ College of Physicians and Surgeons, Columbia University \\ David S. Glenwick \\ Fordham University \\ Yaakov Stern and Thomas K. Tatemichi \\ College of Physicians and Surgeons, Columbia University
}

\begin{abstract}
Sex differences in the representation of visuospatial functions in the human brain were investigated in 20 subjects with right hemisphere stroke and 40 stroke-free control subjects with the Complex Figure Test, WAIS-R Block Design, and Judgment of Line Orientation. The WAIS-R Similarities subtest was administered as a measure of verbal reasoning. The stroke and control groups were composed of equal proportions of males and females, and the male and female stroke groups were matched for location and volume of infarction. A multivariate analysis of covariance determined that the interaction between stroke status and $\operatorname{sex}(p<.05)$, as well as the main effects for stroke status $(p<.001)$ and $\operatorname{sex}(p<.001)$, were significantly related to visuospatial performance. None of these variables was significantly related to WAIS-R Similarities performance. The results of this study suggest that females may be disproportionately impaired in visuospatial functioning relative to males following right hemisphere stroke. We propose that visuospatial functions are bilaterally represented in the brains of males, allowing them to rely upon left hemisphere visuospatial systems following right hemisphere stroke, and that these functions are represented in the right hemisphere of females, resulting in their disproportionate impairment despite comparable lesions.
\end{abstract}

The concept of sex differences in cognitive abilities has provoked much controversy. Numerous studies have been conducted focusing upon this issue, and many theories presented to account for the often conflicting findings (McGlone, 1980). Research with nonclinical populations has demonstrated small but consistent differences favoring males in visuospatial abilities and 
females in verbal abilities (Maccoby \& Jacklin, 1974). In clinical populations, the findings have been difficult to interpret, due to (a) the use of heterogeneous samples, (b) poorly characterized lesions, and (c) the failure to include appropriate comparison groups to better define the "normal" range of performance.

The aim of the present study was to examine sex differences in visuospatial performance in subjects with and without right hemisphere stroke while addressing these methodological issues. In addition, three models of sex differences in the representation of visuospatial functions in the human brain were evaluated.

Model I (Levy, 1980) proposes that visuospatial functions are represented in the right hemisphere of both the mature male brain and the mature female brain, while verbal functions are represented in the left hemisphere of the male brain and in both the left and right hemispheres of the female brain. Model II (Harris, 1978; Inglis, Ruckman, Lawson, MacLean, \& Monga, 1982; Inglis, Ruckman, Lawson, MacLean, \& Monga, 1983; Kimura, 1969; McGlone, 1978) proposes that the mature male brain is more highly lateralized than the mature female brain for visuospatial functions, which are represented in the right hemisphere of the male brain and in both the left and right hemispheres of the female brain. There is disagreement regarding verbal functions, however, which may be represented in the left hemisphere of the male brain and bilaterally in the female brain (Harris, 1978; McGlone, 1978), or in the left hemisphere of both the male and female brains (Inglis et al., 1982; Inglis et al., 1983). Model III (Buffery \& Gray, 1972) proposes that both visuospatial and verbal abilities are bilaterally represented in the male brain, while visuospatial abilities are represented in the right hemisphere and verbal abilities in the left hemisphere of the female brain.

Although somewhat simplistic, these three models provide a conceptual framework for the study of sex differences in cognition. The examination of male and female subjects with comparable brain lesions facilitates this endeavor, as sex-specific patterns of cognitive dysfunction resulting from these lesions might be presumed to result from sex differences in the functional organization of the human brain. The recognition of such differences could have important implications for cognitive rehabilitation following stroke, for it might suggest that sex-specific therapeutic approaches should be utilized to ensure that each patient's recovery is optimal.

\section{METHOD}

\section{Subjects}

The sample consisted of 20 patients with right hemisphere stroke admitted to the stroke unit or general neurology service of Columbia-Presbyterian Medical Center and 40 control subjects without history or clinical evidence of stroke. The stroke and control groups were composed of equal proportions of males and females, and the male and female stroke groups were matched for location and volume of infarction using techniques outlined below. 
Patients with right hemisphere stroke met the following inclusion criteria: (a) 30 days or fewer since onset of ischemic stroke, confirmed by neurological examination and brain imaging; (b) no history of left hemisphere infarction; (c) age between 35 and 85 ; (d) right-handed; (e) adequate visual acuity for visuospatial testing and free of significant hemineglect; ( $f$ ) free of confusion or dementia; and $(\mathrm{g})$ no current substance dependence, as defined in DSM-III-R (American Psychiatric Association, 1987).

Medical inpatients admitted for treatment of orthopedic disorders and individuals randomly selected from Medicare records and a commercial list served as control subjects. They met the same criteria as the patients with right hemisphere stroke but had no history of any neurological disorder that might affect cognition.

\section{Instruments}

Three neuropsychological measures of visuospatial function were selected for use in this study, each of which has proven to be differentially sensitive to right hemisphere lesions:

The Complex Figure Test (CFT: Osterrieth, 1944; Rey, 1940). This measure of spatial organization and constructional skills requires that the subject copy a complex figure from a printed model, with points given for accurate reproduction and placement of the component parts of the figure (Lezak, 1983). Patients with left or right hemisphere stroke are significantly more impaired than control subjects on this measure, and patients with right hemisphere stroke are significantly more impaired than patients with left hemisphere stroke (Binder, 1982). The performance of neurosurgical patients with right temporal lobectomies is significantly poorer than that of patients with left temporal lobectomies (Taylor, 1969).

WAIS-R Block Design (BD: Wechsler, 1981). This subtest of the Wechsler Adult Intelligence Scale-Revised (WAIS-R) requires spatial organization, mental rotation, visuomotor coordination, and constructional skills. The subject must manipulate colored blocks in order to match a series of visually presented designs. Bonus points are awarded for fast performance. Patients with right hemisphere lesions perform more poorly on this measure than those with left hemisphere lesions (Hom \& Reitan, 1990), particularly when the right parietal lobe is involved (Warrington, James, \& Maciejewski, 1986).

Test of Judgment of Line Orientation (JLO: Benton, Hamsher, Varney, \& Spreen, 1983). In this measure of spatial perception, subjects must select 2 of 11 lines of varied angulation to match two lines presented in each test item. Due to the length of time required for administration of the complete test, only the 15 odd-numbered items were used in this study. However, all five examples were still presented in order to prepare the subject for the task. Patients with disorders affecting the right hemisphere are significantly more impaired on this task than patients with left hemisphere involvement (Benton, Hannay, \& Varney, 1975; Benton, Varney, \& Hamsher, 1978). 
Other measures, such as Raven's Coloured Progressive Matrices (Raven, 1965; Raven, Court, \& Raven, 1984) and the Hooper Visual Organization Test (Western Psychological Services, 1983), were not included in this battery because past research has failed to consistently demonstrate that they are differentially sensitive to right hemisphere dysfunction (Boyd, 1981; Drebing, Takushi, Tanzy, Murdock, Stewart, \& Majovsky, 1990; Wang, 1977). The three measures that were selected, however, tap the elementary components of visuospatial functioning (Kritchevsky, 1988), which include spatial constructional ability (e.g., the CFT); spatial mental operations, or mental rotation skills (e.g., BD); spatial perceptual skills, or the ability to analyze the orientation of stimuli and localize those stimuli in space (e.g., JLO); and spatial attentional skills, or the ability to attend to both the left and right hemispaces (all three measures).

WAIS-R Similarities (Wechsler, 1981). In addition to the visuospatial tasks, the Similarities subtest of the WAIS-R was administered in order to examine right hemisphere stroke and sex as potential correlates of verbal reasoning skills. In this task, the subject is presented with pairs of words and asked to define the primary characteristic held in common by each pair of words. Left hemisphere lesions are associated with defective performance on this subtest (Hom \& Reitan, 1990; Warrington et al., 1986).

\section{Procedures}

Prior to inclusion in the study, each subject was screened for hemineglect and deficient visual acuity. A cancellation task was used to exclude subjects with neglect syndromes which might interfere with their ability to attend to the full extent of the test stimuli. Near-vision was assessed with a hand-held eye chart, and a visual acuity of $20 / 100$ or better, with or without corrective lenses, was considered acceptable. If patients were unable to pass these screening tests during the initial evaluation session, they received repeat evaluations every two days. If it was later found that the patient was free of significant visual neglect and visual acuity was adequate for visuospatial testing, the test battery would be administered.

All potential subjects were also screened for dementia. Seven male and seven female stroke patients and 10 male and 11 female control subjects were administered a comprehensive battery of neuropsychological tests (Stern, Andrews, Pittman, Sano, Tatemichi, Lantigua, \& Mayeux, 1992) designed to permit the diagnosis of dementia based upon modified DSM-III-R (American Psychiatric Association, 1987) criteria as part of their participation in a separate study of stroke and dementia (Tatemichi, Desmond, Mayeux, Paik, Stern, Sano, Remien, Williams, Mohr, Hauser, \& Figueroa, 1992). The remaining subjects not enrolled in that study were administered the same battery of tests if any signs of cognitive dysfunction were noted by the clinician or recorded in the subject's medical records. The use of this screening technique resulted in the exclusion of two patients from the present study due to a diagnosis of dementia, while no control subjects were excluded. 
The CT scan of each patient with right hemisphere stroke was analyzed to determine the location and volume of infarction. Infarct location was classified as cortical, cortical/subcortical, or subcortical. In addition, using a variation of a technique described by Turkheimer, Yeo, Jones, and Bigler (1990), the anterior and posterior boundaries of the infarction were quantified as the ratios of the distances from the furthest anterior and posterior extensions of the lesion to the occipital pole relative to the length of the longest slice. Anterior-posterior position was defined as the mean of the furthest anterior and the furthest posterior extensions of the infarction, this value falling within a range of 0 to 1 , with higher values representing a further anterior position.

Infarct volume was calculated using a technique developed for the Stroke Data Bank (Foulkes, Wolf, Price, Mohr, \& Hier, 1988). Infarctions were traced onto a template representing nine axial CT scan slices. A transparent overlay with a reproduction of the nine template slices and a superimposed grid system was then placed upon the infarct tracing and the total number of grid cells occupied by the infarct was calculated. This figure was then multiplied by 1.822 ml., the normative mean volume per grid cell, in order to determine the total volume of the infarction.

Patients in the male and female stroke groups were selected using a pairmatching technique for location of infarction (i.e., cortical, cortical/subcortical, subcortical) and a nearest available matching technique for anterior-posterior position and volume of infarction from an initial sample of 40 patients (Anderson, Auquier, Hauck, Oakes, Vandaele, Weisberg, Bryk, \& Kleinman, 1980).

\section{RESULTS}

An analysis of variance was performed to investigate between-group differences in age and educational background. No significant differences were obtained, but the female stroke group was slightly older and less educated than the other three groups.

Because the male and female patients were selected using a pair-matching technique for location of infarction, no between-group comparison was necessary regarding this variable. $T$-tests were performed to determine whether the male and female stroke groups differed in the anterior-posterior position of infarction, volume of infarction, or the time between onset of stroke and neuropsychological assessment. No significant differences were obtained, and the mean values for the male and female stroke groups were virtually identical for each of these variables. Descriptive statistics for the stroke and control groups are presented in Table 1. Descriptive statistics for the stroke and control groups stratified by sex are presented in Table 2.

A multivariate analysis of covariance was performed, with CFT total score, BD scaled score, and JLO total score as dependent variables, and stroke status, sex, and the interaction between stroke status and sex as independent variables. Age and education were used as covariates because the female stroke group was slightly, although not significantly, older and less educated than the other three groups. 
Table 1. Descriptive Statistics and Test Performance-Stroke and Control Groups

\begin{tabular}{lcc}
\hline \multicolumn{1}{c}{ Variable } & Stroke $(n=20)$ & Control $(n=40)$ \\
\hline Age & $65.0 \pm 12.5$ & $61.8 \pm 14.1$ \\
Education & $12.4 \pm 3.6$ & $14.0 \pm 3.4$ \\
Similarities-scaled score & $7.7 \pm 2.9$ & $9.3 \pm 2.7$ \\
Complex figure test-total score & $20.4 \pm 10.2$ & $31.3 \pm 4.8$ \\
Block design-scaled score & $4.4 \pm 2.6$ & $7.0 \pm 2.4$ \\
Judgment of line orientation & $7.0 \pm 4.9$ & $10.9 \pm 3.6$ \\
\hline
\end{tabular}

Note. Values presented are means \pm one standard deviation.

Table 2. Descriptive Statistics and Test Performance-Stroke and Control Groups Stratified by Sex

\begin{tabular}{|c|c|c|c|c|}
\hline Variable & $\begin{array}{l}\text { Male } \\
\text { Stroke } \\
(n=10)\end{array}$ & $\begin{array}{l}\text { Female } \\
\text { Stroke } \\
(n=10)\end{array}$ & $\begin{array}{c}\text { Male } \\
\text { Control } \\
(n=20)\end{array}$ & $\begin{array}{l}\text { Female } \\
\text { Control } \\
(n=20)\end{array}$ \\
\hline$\overline{\text { Age }}$ & $62.6 \pm 10.6$ & $67.4 \pm 14.3$ & $62.5 \pm 13.2$ & $61.1 \pm 15.3$ \\
\hline Education & $13.2 \pm 4.0$ & $11.5 \pm 3.2$ & $14.2 \pm 3.3$ & $13.8 \pm 3.7$ \\
\hline Days post stroke & $17.4 \pm 8.5$ & $19.5 \pm 8.4$ & na & na \\
\hline Infarct volume (ml.) & $35.1 \pm 32.8$ & $34.9 \pm 49.1$ & na & na \\
\hline \multicolumn{5}{|l|}{ Infarct location } \\
\hline cortical $(\%)$ & 20.0 & 20.0 & na & na \\
\hline cortical/subcortical (\%) & 50.0 & 50.0 & na & na \\
\hline subcortical $(\%)$ & 30.0 & 30.0 & na & na \\
\hline $\begin{array}{l}\text { anterior-posterior } \\
\text { position }\end{array}$ & $.47 \pm .17$ & $.54 \pm .17$ & na & na \\
\hline Similarities-scaled score & $8.7 \pm 3.1$ & $6.7 \pm 2.4$ & $9.3 \pm 3.0$ & $9.3 \pm 2.5$ \\
\hline Complex figure test & & & & \\
\hline $\begin{array}{l}\text { - total score } \\
\text { Block design }\end{array}$ & $26.4 \pm 9.3$ & $14.4 \pm 7.2$ & $31.9 \pm 4.4$ & $30.7 \pm 5.1$ \\
\hline -scaled score & $5.8 \pm 2.5$ & $3.0 \pm 1.9$ & $7.1 \pm 2.5$ & $7.0 \pm 2.4$ \\
\hline $\begin{array}{l}\text { Judgment of } \\
\text { line orientation }\end{array}$ & $10.5 \pm 3.5$ & $3.4 \pm 3.2$ & $12.2 \pm 2.3$ & $9.7 \pm 4.3$ \\
\hline
\end{tabular}

Note. Values presented are means \pm one standard deviation.

The interaction between stroke status and sex was significant, $F(3,52)=3.10$, $p<.05$. Univariate tests were significant for CFT, $F(1,54)=8.85, p<.01$, and JLO total scores, $F(1,54)=4.82, p<.05$, and a trend was noted for BD scaled score, $F(1,54)=3.16, p<.10$. Scheffe post hoc comparisons determined that the female stroke group exhibited the poorest visuospatial performance of the four groups. Test results are presented in Table 1 for the stroke and control groups and in Table 2 for the stroke and control groups stratified by sex.

The main effect for sex was also significant, $F(3,52)=9.58, p<.001$. Univariate tests were significant for CFT, $F(1,54)=13.74, p<.001$, and JLO total scores, $F(1,54)=24.85, p<.001$, and a trend was noted for BD scaled score, $F(1,54)=3.74, p<.10$, with mean scores favoring male patients compared to female patients and male control subjects compared to female control subjects. 
Finally, the main effect for stroke status was significant, $F(3,52)=12.01$, $p<.001$, favoring control subjects. Univariate tests were significant for every visuospatial measure, including the CFT, $F(1,54)=36.17, p<.001, \mathrm{BD}$, $F(1,54)=12.06, p<.01$, and JLO, $F(1,54)=14.57, p<.01$.

With respect to the WAIS-R Similarities subtest, an analysis of covariance was performed, with scaled score on this subtest as the dependent variable; stroke status, sex, and the interaction between stroke status and sex as independent variables; and age and education as covariates. None of the independent variables was significantly related to WAIS-R Similarities performance.

\section{DISCUSSION}

The results of this study suggest that females may be disproportionately impaired in visuospatial functioning relative to males following right hemisphere stroke. Previous studies have reported inconsistent results. Some studies that have administered the Wechsler-Bellevue I, WAIS, or WAIS-R, or specific subtests from those batteries, to patients with right hemisphere lesions found that males exhibited greater deficits than females in nonverbal performance (Bornstein \& Matarazzo, 1982; Inglis \& Lawson, 1981; Inglis et al., 1983; Lewis \& Kamptner, 1987; Sundet, 1986); other studies did not find a significant sex difference using similar assessment procedures (Bornstein, 1984; McGlone, 1978; Snow \& Sheese, 1985; Warrington et al., 1986; Yeo, Turkheimer, \& Bigler, 1984). In studies using measures not taken from the Wechsler batteries in the assessment of patients with right hemisphere lesions, males received significantly higher scores than females on the Mooney Closure Faces Test (Lansdell, 1968), but significant sex differences were not obtained with the Complex Figure Test (Hier \& Kaplan, 1980), Raven's Coloured Progressive Matrices (McGlone \& Kertesz, 1973), the Street Gestalt Completion Test (Lewis \& Kamptner, 1987), or a tachistoscopically presented line orientation task (Benton et al., 1975).

A significant methodological weakness of these studies, however, lies in their inclusion of patients with a variety of lesion types (e.g., stroke and tumor) and/or their inadequate characterizations of those lesions. The single study that utilized advanced methods to quantify lesion volume and location included patients with ischemic stroke, hemorrhage, tumor, trauma, and abscess (Yeo et al., 1984), significantly diminishing the value of the use of those methods. Those studies that attempted to examine patients with a specific lesion type provided little, if any, information other than the laterality of the involved hemisphere. A significant between-group difference in lesion type, and, in the case of lateralized lesions, site or size, could have been the sole basis for the greater visuospatial impairment that has been observed in males following unilateral brain damage in some studies or the lack of significant results in other studies. Without more explicit information regarding lesion characteristics, the findings of prior studies should be interpreted with caution. 
In contrast, the results of our study were not confounded by sex differences in lesion characteristics because patients with hemispheral lesions other than stroke were excluded, and male and female patients were well matched for volume and location of infarction. Therefore, the most likely remaining explanation for the significant interaction between stroke status and sex would be a difference between males and females in the functional organization of the brain.

The results of our study permit a critical examination of the three models of sex differences in the functional organization of the human brain that were described above. Model I proposed that visuospatial functions are represented in the right hemisphere of both males and females, with the right hemisphere of females also being partially dedicated to language functions. This model might suggest that males and females would be equally affected by right hemisphere stroke. Given the asymmetrical representation of visuospatial functions in males and females proposed by this model, damage to the right hemisphere could produce a comparable degree of decline in the performance of both groups, with the male superiority in visuospatial functions recognized in subjects without brain damage remaining evident and of equal magnitude in the stroke group. As evidenced by the significant Stroke Status $x$ Sex interaction, our results are not consistent with that prediction.

Model I also proposed that the recognized male superiority in visuospatial functioning is the result of a right hemisphere that is fully dedicated to those functions, allowing males more processing power for visuospatial tasks. Greater visuospatial dysfunction in females following right hemisphere stroke might result from damage to two processing systems, with disruption of right hemisphere verbal systems potentially compromising the ability of females to verbally mediate their performance in visuospatial tasks, a sex-specific strategy suggested by earlier studies (Kimura, 1969). The WAIS-R Similarities subtest, a measure of verbal reasoning, was also administered in this study and can provide useful information regarding this issue. Significant between-group differences in performance on this test were not obtained for stroke status, sex, or for the interaction between stroke status and sex. This would suggest that verbal reasoning was not differentially impaired in females following right hemisphere stroke, and that defective verbal mediation was not the basis for poorer visuospatial performance in females.

Model II suggested that visuospatial functions are bilaterally represented in females but asymmetrically represented in the right hemisphere of males. This model might suggest that females could rely upon left hemisphere visuospatial systems following right hemisphere stroke, allowing them to function at a level equal to or better than males in the visuospatial domain. This prediction is also inconsistent with our findings.

The results of this study are most consistent with Model III. Visuospatial functions may be represented in the right hemisphere of females, as suggested by Model I, but bilaterally represented in males, allowing males to rely upon left hemisphere visuospatial systems following right hemisphere damage. This would, thus, allow males to function more effectively than females in the visuospatial domain following right hemisphere stroke. Bilateral representation 
might be optimal for visuospatial functions because it would provide the substrate for evenly distributed attention to both the left and right hemispaces, thereby eliminating any location-based bias that might compromise the higherlevel processing of nonverbal stimuli that are scattered randomly throughout the environment.

Our findings have additional implications related to the independent effects of right hemisphere stroke and sex on visuospatial functioning. Stroke status was significantly related to performance on each of the visuospatial tasks, with the control group receiving higher scores on every measure, but it was not significantly related to verbal reasoning skills as assessed with the WAIS-R Similarities subtest. These findings are in accord with those of many other studies that have recognized the right hemisphere as being of primary importance in visuospatial functioning (Benton, 1985). Sex was significantly related to CFT and JLO performance and demonstrated a nonsignificant trend in its relationship to $\mathrm{BD}$ performance. Mean scores tended to favor male patients relative to female patients and male control subjects relative to female control subjects on each of the visuospatial measures. These findings are consistent with those of many other studies that have reported a male superiority in the visuospatial domain (Maccoby \& Jacklin, 1974; McGlone, 1980).

Our study hascertain limitations. Although our results suggest that visuospatial functions may be represented bilaterally in the male brain, we did not study patients with left hemisphere lesions. Future studies should examine sex differences in visuospatial performance following left hemisphere stroke to determine whether males would be disproportionately impaired in this domain. It has been suggested that a sex difference may exist in the intrahemispheric representation of certain cognitive functions (Kimura, 1983), and this should be an additional focus of future studies. Ideally, larger samples should be examined, but these can be difficult to obtain when strict enrollment criteria and matching techniques are being utilized in order to eliminate the potentially confounding effects of many other variables. Other methodologies might also provide valuable information regarding sex differences in brain organization, such as functional imaging with positron emission tomography (PET) or single photon emission computed tomography (SPECT). Studies of these types could be used to determine whether stimulation in different modalities (e.g., verbal vs. nonverbal) would provoke sex-specific patterns of brain activation.

Finally, we suggest that future studies should investigate sex differences in the efficacy of rehabilitation techniques, particularly those that may influence cognitive performance. Given that males and females may exhibit different patterns of cognitive dysfunction despite comparable brain lesions, it is possible that the use of sex-specific rehabilitation techniques would help to ensure optimal recovery following stroke. For example, the rehabilitation of female patients with hemineglect following right hemisphere stroke might emphasize their use of verbal mediation and self-prompting (e.g., "Look left") to overcome spatial inattention through head repositioning. In contrast to the technique used with females, head repositioning in males might be encouraged through comput- 
erized retraining in which the patient is exposed to nonverbal stimuli in different positions on a computer screen and lateralized auditory cues are used to direct the patient's attention to those stimuli.

\section{REFERENCES}

American Psychiatric Association. (1987). Diagnostic and statistical manual of mental disorders (3rd ed., revised). Washington, DC: Author.

Anderson, S., Auquier, A., Hauck, W.W., Oakes, D., Vandaele, W., Weisberg, H.I., Bryk, A.S., \& Kleinman, J. (1980). Statistical methods for comparative studies: Techniques for bias reduction. New York: Wiley.

Benton, A. (1985). Visuoperceptual, visuospatial, and visuoconstructive disorders. In K. M. Heilman \& E. Valenstein (Eds.), Clinical neuropsychology (2nd ed., pp. 151-185). New York: Oxford University Press.

Benton, A. L., Hamsher, K., Varney, N. R., \& Spreen, O. (1983). Contributions to neuropsychological assessment: A clinical manual. New York: Oxford University Press.

Benton, A. L., Hannay, H. J., \& Varney, N. R. (1975). Visual perception of line direction in patients with unilateral brain disease. Neurology, 25, 907-910.

Benton, A. L., Varney, N. R., \& Hamsher, K. (1978). Visuospatial judgment: A clinical test. Archives of Neurology, 35, 364-367.

Binder, L.M. (1982). Constructional strategies on complex figure drawings after unilateral brain damage. Journal of Clinical Neuropsychology, 4, 51-58.

Bornstein, R. A. (1984). Unilateral lesions and the Wechsler Adult Intelligence Scale-Revised: No sex differences. Journal of Consulting and Clinical Psychology, 52, 604-608.

Bornstein, R. A., \& Matarazzo, J. D. (1982). Wechsler VIQ versus PIQ differences in cerebral dysfunction: A literature review with emphasis on sex differences. Journal of Clinical Neuropsychology, 4, 319-334.

Boyd, J. L. (1981). A validity study of the Hooper Visual Organization Test. Journal of Consulting and Clinical Psychology, 49, 15-19.

Buffery, A. W. H., \& Gray, J. A. (1972). Sex differences in the development of spatial and linguistic skills. In C. Ounsted \& D. Taylor (Eds.), Gender differences, their ontogeny and significance (pp. 123-158). Edinburgh: Churchill Livingstone.

Drebing, C. E., Takushi, R. Y., Tanzy, K .S., Murdock, G. A., Stewart, J. C., \& Majovsky, L. V. (1990). Re-examination of CPM performance and neglect in lateralized brain injury. Cortex, 26, 661-664.

Foulkes, M. A., Wolf, P. A., Price, T. R., Mohr, J. P., \& Hier, D. B. (1988). The Stroke Data Bank: Design, methods, and baseline characteristics. Stroke, 19 , 547-554.

Harris, L. J. (1978). Sex differences in spatial ability: Possible environmental, genetic, and neurological factors. In M. Kinsbourne (Ed.), Asymmetrical function of the brain (pp.405-522). New York: Cambridge University Press. 
Hier, D. B., \& Kaplan, J. (1980). Are sex differences in cerebral organization clinically significant? Behavioral and Brain Sciences, 3, 238-239.

Hom, J., \& Reitan, R. M. (1990). Generalized cognitive function after stroke. Journal of Clinical and Experimental Neuropsychology, 12, 644-654.

Inglis, J., \& Lawson, J. S. (1981). Sex differences in the effects of unilateral brain damage on intelligence. Science, 212, 693-695.

Inglis, J., Ruckman, M., Lawson, J.S., MacLean, A. W., \& Monga, T. N. (1982). Sex differences in the cognitive effects of unilateral brain damage. Cortex, $18,257-275$.

Inglis, J., Ruckman, M., Lawson, J. S., MacLean, A. W., \& Monga, T. N. (1983). Sex differences in the cognitive effects of unilateral brain damage: Comparison of stroke patients and normal control subjects. Cortex, 19, 551-555.

Kimura, D. (1969). Spatial localization in left and right visual fields. Canadian Journal of Psychology, 23, 445-458.

Kimura, D. (1983). Sex differences in cerebral organization for speech and praxic functions. Canadian Journal of Psychology, 37, 19-35.

Kritchevsky, M. (1988). The elementary spatial functions of the brain. In J. StilesDavis, M. Kritchevsky \& U. Bellugi (Eds.), Spatial cognition: Brain bases and development (pp. 111-140). Hillsdale, NJ: Lawrence Erlbaum Associates.

Lansdell, H. (1968). Effect of extent of temporal lobe ablations on two lateralized deficits. Physiology and Behavior, 3, 271-273.

Levy, J. (1980). Cerebral asymmetry and the psychology of man. In M.C. Wittrock (Ed.), The brain and psychology (pp. 245-321). New York: Academic Press.

Lewis, R. S., \& Kamptner, N. L. (1987). Sex differences in spatial task performance of patients with and without unilateral cerebral lesions. Brain and Cognition, 6, 142-152.

Lezak, M. D. (1983). Neuropsychological assessment (2nd ed.). New York: Oxford University Press.

Maccoby, E. M., \& Jacklin, C. N. (1974). The psychology of sex differences. Stanford, CA: Stanford University Press.

McGlone, J. (1978). Sex differences in functional brain asymmetry. Cortex, 14, 122-128.

McGlone, J. (1980). Sex differences in human brain asymmetry: A critical survey. Behavioral and Brain Sciences, 3, 215-263.

McGlone, J., \& Kertesz, A. (1973). Sex differences in cerebral processing of visuospatial tasks. Cortex, 9, 313-320.

Osterrieth, P. A. (1944). Le test de copie d'une figure complexe [The complex figure copying test]. Archives de Psychologie, 30, 206-356.

Raven, J.C. (1965). Guide to using the Coloured Progressive Matrices. London: H.K. Lewis.

Raven, J. C., Court, J. H., \& Raven J. (1984). Manual for Raven's Progressive Matrices and Vocabulary Scales. London: H.K. Lewis.

Rey, A. (1940). L'examen psychologique dans les cas d'encephalopathie traumatique [The psychological evaluation in cases of traumatic encephalopathy]. Archives de Psychologie, 28, 286-340. 
Snow, W. G., \& Sheese, S. (1985). Lateralized brain damage, intelligence, and memory: A failure to find sex differences. Journal of Consulting and Clinical Psychology, 53, 940-941.

Stern, Y., Andrews, H., Pittman, J., Sano, M., Tatemichi, T., Lantigua, R., \& Mayeux, R. (1992). Diagnosis of dementia in a heterogeneous population: Development of a neuropsychological paradigm-based diagnosis of dementia and quantified correction for the effects of education. Archives of Neurology, $49,453-460$.

Sundet, K. (1986). Sex differences in cognitive impairment following unilateral brain damage. Journal of Clinical and Experimental Neuropsychology, 8, 51-61.

Tatemichi, T. K., Desmond, D. W., Mayeux, R., Paik, M., Stern, Y., Sano, M., Remien, R.H., Williams, J. B. W., Mohr, J. P., Hauser, W. A., \& Figueroa, M. (1992). Dementia after stroke: Baseline frequency, risks, and clinical features in a hospitalized cohort. Neurology, 42, 1185-1193.

Taylor, L. B. (1969). Localisation of cerebral lesions by psychological testing. Clinical Neurosurgery, 16, 269-287.

Turkheimer, E., Yeo, R. A., Jones, C. L., \& Bigler, E. D. (1990). Quantitative assessment of covariation between neuropsychological function and location of naturally occurring lesions in humans. Journal of Clinical and Experimental Neuropsychology, 12, 549-565.

Wang, P. L. (1977). Visual organization ability in brain-damaged adults. Perceptual and Motor Skills, 45, 723-728.

Warrington, E. K., James, M., \& Maciejewski, C. (1986). The WAIS as a lateralizing and localizing diagnostic instrument: A study of 656 patients with unilateral cerebral lesions. Neuropsychologia, 24, 223-239.

Wechsler, D. (1981). Manual for the Wechsler Adult Intelligence Scale-Revised. New York: The Psychological Corporation.

Western Psychological Services. (1983). The Hooper Visual Organization Test. Los Angeles: Author.

Yeo, R. A., Turkheimer, E., Bigler, E. D. (1984). The influence of sex and age on unilateral cerebral lesion sequelae. International Journal of Neuroscience, 24, 299-301.

Acknowledgments. This work was supported in part by Grants R01-NS26179 and P01AG07232 and Clinical Investigator Development Award K08-NS01635 (Dr. Desmond) from the National Institutes of Health.

Offprints. Requests for offprints should be sent to Dr. Desmond, Neurological Institute, 710 West 168th Street, New York, NY 10032.

Submitted: April 1993

Revised: June 1993

Accepted: July 1993 